\title{
ERRATUM
}

Christoph Röcken · Beate Eick - Wolfgang Saeger

\section{Senile amyloidoses of the pituitary and adrenal glands}

\section{Morphological and statistical investigations}

Virchows Arch (1996) 429:293-299

Figure 3 does not show immunostaining of interstitial pituitary amyloid with anti-A-lambda (HAR), but rather immunostaining of the adrenal capsule with anti-Alambda (HAR) ( 2 cases), which was not related to amyloid deposits. 